\title{
A Case Report of Fever of Unknown Origin (FUO)
}

\author{
Shuang Huang*, Yonghong Zhang
}

Hematology Department, Beijing Children’s Hospital, Beijing, China.

Email: *huangshuangd125@163.com

Received May $19^{\text {th }}, 2013$; revised June $22^{\text {nd }}, 2013$; accepted July $1^{\text {st }}, 2013$

Copyright @ 2013 Shuang Huang, Yonghong Zhang. This is an open access article distributed under the Creative Commons Attribution License, which permits unrestricted use, distribution, and reproduction in any medium, provided the original work is properly cited.

\begin{abstract}
A 10-year-old boy had a long time of fever, and was diagnosed as JRA at first, but the patient's condition got worse and worse after the treatment, then we did the cervical lymph node biopsy, which showed ALCL (Anaplastic Large Cell Lymphoma). After receiving the correct treatment, the patient's condition got better.
\end{abstract}

Keywords: FUO; ALCL; Misdiagnose

\section{Introduction}

Fever of unknown origin (FUO) refers to a condition in which the patient has an elevated temperature, but no explanation has been found in spite of investigations by a physician. The cause is found by eliminating all possibilities until only one explanation remains, and taking this as the correct one, it is usually a diagnosis of exclusion.

\section{Report of a Case}

A 10-year-old boy, with a 3-month history of fever, without any other simultaneous complications, antibiotics were ineffective.

Physical examination showed vital signs were stable, conscious, no pallor, no lymphadenopathy, heart and lungs were normal in auscultation, liver and spleen were just palpable below the costal margins, no joint swelling nervous system examinations were normal.

Lab results: CBC: WBC $15 \times 10^{9} / \mathrm{L}, \mathrm{N} 84 \%$, Hb 110 g/L, PLT $450 \times 10^{9} / \mathrm{L}$, CRP $120 \mathrm{mg} / \mathrm{L}$, serum ferritin: 1500 ug/L, LDH: 550 IU/L, AST 45 IU/L, ALT 18 IU/L, PPD: (-), blood cultures: no bacteria and fungus, virus antibodies: EBV, CMV were negative, autoantibody screening: negative, bone marrow aspiration: normal cellularity, no tumor cells. Abdominal ultrasound: enlarged abdominal lymph nodes and splenohepatomegal chest X-ray: normal, cranial CT and brain MRI scan: normal.

Impression: FUO (1) JRA? (2) Infections?

Treatment and the change of the patient's conditions

*Corresponding author. are as follows:

In the 1st week, the doctor of local hospital gave the patient prednisone to take in, the temperature became normal for a few days, but relapsed soon, the liver and spleen enlarged a lot. In the 2nd week, the patient's fever peak increased, it reached to $40^{\circ} \mathrm{C}$, the WBC and CRP, ESR were all increased, the doctor gave the patient ibuprofen for treatment, but it was still useless. In the 3rd week, the patient had a very sever cough and dyspnea, the cervical lmph node enlarged, the doctor gave him nimesulide to control the temperature, and transferred the patient to our hospital, and we did the cervical lymph node biopsy by needle core (Figures 1-3), and the pulmonary CT scan (Figure 4), after that, we diagnosed the patient as ALCL (Anaplastic Large Cell Lymphoma), the involvement included cervical lymph node, mediastinum, liver, and spleen. In the 4th week, we gave the patient chemotherapy for treatment, the condition of the patient became better soon, and we did the pulmonary CT scan again (Figure 5).

\section{Discussion}

In 1961 Petersdorf and Beeson suggested the following criteria of FU0 [1]:

1) Fever higher than $38.3^{\circ} \mathrm{C}\left(101^{\circ} \mathrm{F}\right)$ on several occasions;

2) Persisting without diagnosis for at least 3 weeks;

3) At least 1 week's investigation in hospital.

A new definition which includes the outpatient setting (which reflects current medical practice) is broader, stipulating: (1) outpatient visits or 3 days in the hospital 


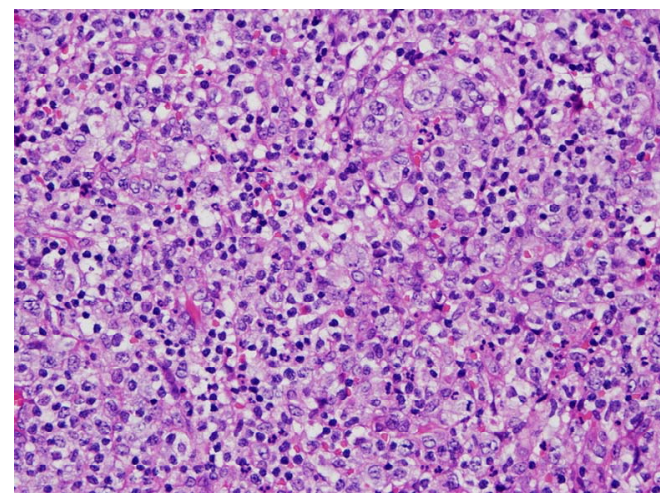

Figure 1. HE staining of cervical lymph node.

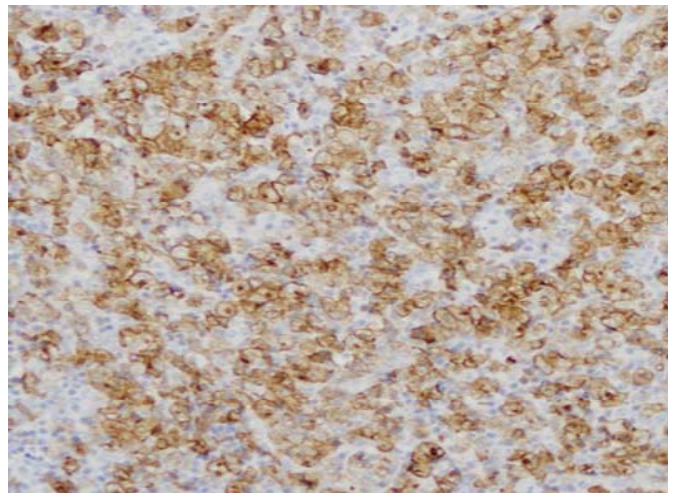

Figure 2. CD30 IHC was positive.

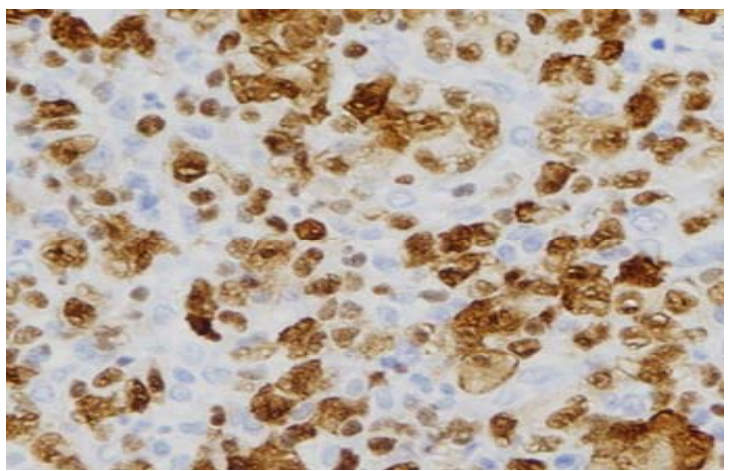

Figure 3. ALK staining was positive.

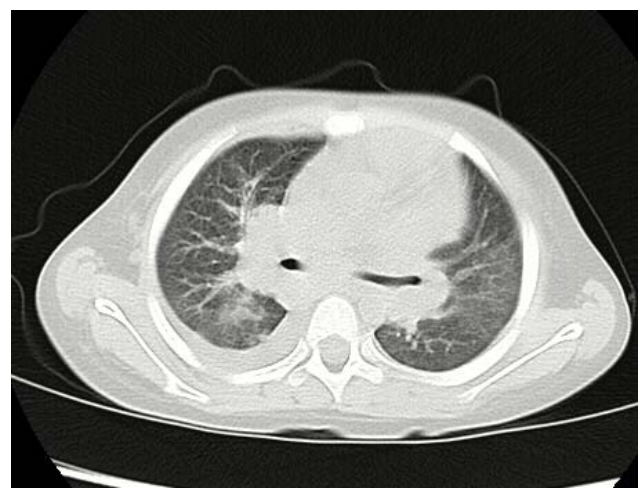

Figure 4. Pulmonary CT scan before treatment.

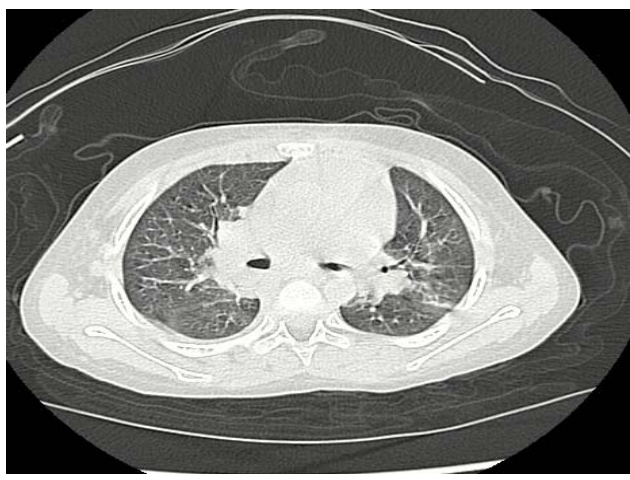

Figure 5. Pulmonary CT scan after treatment.

without elucidation of a cause or 1 week of "intelligent and invasive" ambulatory investigation. The classifications of FUO are: 1) infections (e.g. abscesses, endocarditis, tuberculosis, and complicated urinary tract infections); 2) malignancy (e.g. lymphoma, leukaemias); 3) connective tissue diseases (e.g. systemic lupus erythematosus, and rheumatoid arthritis); 4) miscellaneous disorders (e.g. alcoholic hepatitis, granulomatous conditions); 5) undiagnosed conditions [1,2].

As we know, the paediatric non-Hodgkin lymphomas (NHL) are a diverse collection of lymphoid malignancies with varied pathologies, cells of origin, natural history, and response to treatment. The histrionic diagnosis of NHL is among the most difficult tasks that surgical pathologists are asked to undertake. A general diagnosis of NHL is accurate in roughly $90 \%$ of cases; however, consensus among pathologists regarding the identification of specific histologic subtypes occurs only slightly more than half of the time in past studies using morphology only. Integration of morphologic, immunophenotypic, and genetic information into classifications of NHL has invaluable in providing the basis for our current approaches to the diagnosis and therapeutic management of theses malignancies by providing a framework to subdivide the disease into clinically meaningful and diagnostically reproducible subgroups. Refinements in theses classification schemes are constantly ongoing as additional parameters are identified and shown to permit the detection of subgroups that possess unique biologic features and/or that have either a specific response to traditional therapies or are amenable to more directed forms of treatment. Non-Hodgkin lymphoma comprise approximately $10 \%$ of all childhood cancers and are a diverse collection of malignant neoplasm of lymphoreticular cells. Paediatric NHL includes a varied group of neoplasms that derive from both mature and immature (blastic) cells of both B-cell and T-cell origin. ALCL is the most common mature T-cell lymphoma seen in children and makes up approximately $10 \%$ of all paediatric NHL and approximately $30 \%-40 \%$ of the large-cell lymphomas seen in the paediatric population [3]. 
ALCL was first described by Stein et al. in 1985.This new entity was characterized by large anaplastic cells with strong reactivity to the monoclonal antibody, Ki-1 (CD30). Morphologically, primary systemic ALCL can be quite variable. Classic ALCL has a predominance of tumor cells that are large, pleomorphic, and often multinucleated. Often these cells contain eccentric horseshoeshaped nuclei with abundant clear-to-basophilic cytoplasm with an area of eosinophilia near the nucleus. A small-cell variant of ALCL has also been described where the cells are smaller in size, more monomorphic, and show minimal cytologic variation. ALCL has tumor cells which express the CD30 (ki-1) antigen in virtually all cases. The majority of tumors will have T-cell antigens $[4,5]$ The clinical presentation of children and adolescents with ALCL is characterized by several distinctive features. Extronodal involvement including skin, bone, soft tissue, and the presence of B-symptoms are more frequent. Patients may have an indolent phase consisting of lymphadenopathy or a longer illness characterized by fever and a long time of lymphadenopathy before diagnosis.

We reviewed the lymphoma patients from 2003 to 2008 in Beijing Children's Hospital and found that 133 out of 227 patients had been misdiagnosed with other diseases at first. The patients were divided into different groups by pathological study (LBL, HL, ALCL, mature B-cell lymphoma) and we found that ALCL patients' misdiagnosis rate was the highest, reaching up to $93.3 \%$. The most common reasons are:

1) The presence of extranodal involvement and various initial symptoms make clinical doctors do not have enough understandings of lymphoma.

2) Pathologists do not have enough of an understanding of ALCL and some samples are not integrated or correctly disposed.

3) Some parents of patients are noncompliant. About $12.8 \%$ of patients were delayed diagnosis.

4) Inappropriate usage of steroids before diagnosis caused some patients to be misdiagnosed.

5) Some cases were misdiagnosed because of the rare clinical manifestation.

\section{Conclusion}

In conclusion, the clinical manifestations of ALCL are various, and the extranodal involvement is more common than other lymphomas, so the patients are misdiagnosed easily as other diseases, such as infection disease, CTD, or others. So when you meet a FUO patient, if his/her conditions are not better after a long time treatment, a new assessment should be done, and the possibility of ALCL should be considered too.

\section{REFERENCES}

[1] D. C. Norman, M. B. Wong and T. T. Yoshikawa, "Fever of Unknown Origin in Older Persons," Infectious Disease Clinics of North America, Vol. 21, No. 4, 2007, pp. 937945. doi:10.1016/j.idc.2007.09.003

[2] H. A. Majeed, "Differential Diagnosis of Fever of Unknown Origin in Children," Current Opinion in Rheumatology, Vol. 12, No. 5, 2000, pp. 439-444. doi:10.1097/00002281-200009000-00016

[3] A. Takami, H. Okumura, Y. Maeda, Y. Kumano, H. Asakura, M. Oda, K. Omura and S. Nakao, "Primary Tracheal Lymphoma: Case Report and Literature Review," International Journal of Hematology, Vol. 82, No. 4, 2005, pp. 338-342. doi:10.1532/IJH97.05087

[4] M. M. Tomaszewski, J. C. Moad and G. P. Lupton, "Primary Cutaneous Ki-1(CD30) Positive Anaplastic Large Cell Lymphoma in Childhood," Journal of the American Academy of Dermatology, Vol. 40, No. 5, 1999, pp. 857861. doi:10.1053/jd.1999.v40.a95960

[5] K. Khodadad, "Primary Anaplastic Large Cell Lymphoma of Trachea with Subcutaneous Emphysema and Progressive Dyspnea," Hematology/Oncology and Stem Cell Therapy, Vol. 4, No. 4, 2011, pp. 188-191. 